\section{Tendência temporal e fatores determinantes da anemia em crianças de duas faixas etárias (6-23 e 24-59 meses) no Estado de Pernambuco, Brasil, 1997-2006}

\author{
Time trends in anemia and associated factors \\ in two age groups (6-23 and 24-59 months) in \\ Pernambuco State, Brazil, 1997-2006 \\ Tendencia temporal y factores de anemia en \\ niños dentro de dos grupos de edad (6-23 y \\ 24-59 meses) en el estado de Pernambuco, \\ Brasil, 1997-2006
}

\author{
Priscila Nunes de Vasconcelos 1 \\ Débora Silva Cavalcanti 1 \\ Luciana Pedrosa Leal 2 \\ Mônica Maria Osório 2 \\ Malaquias Batista Filho ${ }^{3}$
}

\author{
1 Programa de Pós-graduação \\ em Nutrição, Universidade \\ Federal de Pernambuco, \\ Recife, Brasil. \\ 2 Programa de Pós- \\ graduação em Enfermagem \\ Universidade Federal de \\ Pernambuco, Recife, Brasil. \\ 3 Instituto de Medicina \\ Integral Prof. Fernando \\ Figueira, Recife, Brasil. \\ Correspondência \\ P. N. Vasconcelos \\ Programa de Pós-graduação \\ em Nutrição, Universidade \\ Federal de Pernambuco. \\ Av. Brigadeiro Eduardo \\ Gomes 1205, Maceió, AL \\ 57038-230, Brasil. \\ priscilanv@msn.com
}

\begin{abstract}
Data from two health and nutrition surveys were used to analyze times trends in anemia and associated factors in children 6-23 and 2459 months of age in Pernambuco State, Brazil. The samples totaled 777 and 993 children 6-59 months of age in the 2nd PESN/1997 and 3rd PESN/2006 surveys, respectively. The exploratory variables were grouped into five hierarchical levels: socioeconomic factors; economic index and environmental index; maternal factors; health and nutritional care; and morbidity and nutritional status, analyzed by Poisson logistic regression. From the first to the second survey, anemia prevalence decreased by $11.7 \%$ and $33.4 \%$, respectively, for children 6-23 and 24-59 months of age. In the time trend analysis, only the lower tertile of the environmental index remained as a determinant factor for anemia in children 6-23 months of age, and the lower tertile of the economic index remained statistically significant in children 24-59 months of age. The study concludes that the decrease in anemia was more significant in the 24-59 month age group.
\end{abstract}

Time Series Studies; Anemia; Child

\section{Resumo}

Para analisar a tendência temporal da anemia $e$ de fatores associados em crianças de 6-23 e de 24-59 meses no Estado de Pernambuco, Brasil, foram utilizados os dados de dois inquéritos de saúde e nutrição. As amostras totalizaram $777 e$ 993 crianças de 6-59 meses, respectivamente na II PESN/1997 e na III PESN/2006. As variáveis exploratórias foram agrupadas em cinco níveis hierárquicos: fatores socioeconômicos; índice econômico e índice ambiental; fatores maternos; assistência à saúde e nutrição; morbidade e estado nutricional, analisadas pela regressão múltipla de Poisson. Entre os dois inquéritos, as prevalências de anemia apresentaram uma diminuição de 11,7\% e $33,4 \%$, respectivamente, para as crianças de 6-23 e de 24-59 meses. Na análise de tendência temporal apenas o tercil inferior do indice ambiental permaneceu como fator determinante da anemia para as crianças de 6-23 meses, e o tercil inferior do índice econômico se manteve estatisticamente significativo nas crianças de 24-59 meses. Concluise que houve diminuição mais significativa da anemia no grupo de 24-59 meses.

Estudos de Séries Temporais; Anemia; Crianças 


\section{Introdução}

A prevalência dos problemas carenciais da população brasileira nos últimos trinta anos tem apresentado notáveis modificações, assinalando uma rápida mudança na sua ocorrência e distribuição geográfica, temporal e social, caracterizando a chamada transição nutricional. No entanto, a situação das anemias em menores de cinco anos de idade manteve-se estacionária ou em elevação ${ }^{1}$. Tais peculiaridades conferem ao estudo da anemia em nível populacional, sobretudo em crianças, um interesse e atualidade inquestionáveis.

No Brasil, inquéritos nutricionais realizados sucessivamente para representar universos populacionais (cidade de São Paulo e Estado da Paraíba) evidenciaram uma elevação surpreendente na prevalência de anemia em menores de cinco anos, nas décadas de 80 e 90, duplicando ou até triplicando os valores detectados na linha de base destas duas avaliações 2,3. Uma revisão sistemática 4 considera que a prevalência de anemia em crianças brasileiras continua elevada, com variações compreendidas entre $22,2 \%$ e $54 \%$.

Estudos mais recentes 5,6, no entanto, parecem indicar nova tendência, com uma redução em torno de $30 \%$ na prevalência de anemia em crianças. Essa nova situação torna-se singularmente interessante, desde que o cenário epidemiológico das anemias, em nível global, praticamente se mantém inalterado a partir da década de 80 7. Torna-se pertinente considerar que após 2004 parece ter ocorrido uma tendência diferenciada no panorama das anemias no Brasil, notadamente em crianças e mulheres no período reprodutivo 8,9.

Em Pernambuco, a tendência de diminuição da prevalência da anemia tem sido demonstrada na comparação temporal realizada com base nos inquéritos populacionais do estado, observando-se uma diminuição da prevalência de 40,6\%, em 1997, para 32,8\% em 2006, em menores de cinco anos 5 .

Diversos fatores, biológicos, socioeconômicos, ambientais, de saúde e nutrição, podem contribuir para a ocorrência de anemia e a complexidade no seu controle faz com que a Organização Mundial da Saúde (OMS) recomende que estudos de base populacional sejam realizados, a fim de investigar as razões para as altas prevalências e seus potenciais fatores de risco, visando subsidiar novas ações governamentais ${ }^{7}$.

Considerando que na idade de 6-23 meses as crianças apresentam um risco maior de serem anêmicas quando comparadas com as de 24 meses e mais 5,10 , questiona-se as diferenças nos fatores estatisticamente significativos na determinação da anemia entre os dois grupos etários, nos dois contextos temporais da realização dos estudos.

Nessas condições, o Estado de Pernambuco, dispondo de estudos representativos da situação nutricional de sua população na segunda metade da década de 90 (1997) e nove anos após (2006), envolvendo as mesmas instituições de pesquisa e os mesmos procedimentos metodológicos nos dois inquéritos, oferece uma condição apropriada para realizar comparações temporais e, como desdobramento, testar hipóteses que possam figurar na base explicativa das mudanças ocorridas.

Este estudo, portanto, tem o objetivo de verificar a tendência temporal de evolução da anemia e de seus fatores associados em crianças de 6-23 meses e de 24-59 meses, nos anos de 1997 e 2006, no Estado de Pernambuco.

\section{Métodos}

Este trabalho foi desenvolvido utilizando dados originais da II Pesquisa Estadual de Saúde e Nutrição de Pernambuco (II PESN/PE) 11, realizada em 1997, e da III PESN/PE de 2006 12. As duas pesquisas foram realizadas mediante parcerias entre o Departamento de Nutrição da Universidade Federal de Pernambuco, o Instituto de Medicina Integral Prof. Fernando Figueira (IMIP) e a Secretaria Estadual de Saúde de Pernambuco.

O Estado de Pernambuco tem uma área de 98.146,315km² , distribuída em 185 municípios, com uma população estimada de 8.796 .032 habitantes em 2010 (Instituto Brasileiro de Geografia e Estatística. Censo Populacional 2010. http:// censo2010.ibge.gov.br/resultados, acessado em 10/Nov/2013). A população do estado é predominantemente urbana, concentrando $80 \%$ dos habitantes.

Nas pesquisas de 1997 e 2006, utilizou-se uma amostragem probabilística dos dois estratos em que foi dividido o espaço geográfico estadual: urbano e rural. O processo de amostragem foi realizado em três estágios. No primeiro, foram mantidos os mesmos 18 dos 185 municípios sorteados por probabilidade proporcional ao tamanho de sua população no inquérito da I PESN/PE 13. No segundo estágio, tendo como base os municípios previamente selecionados, foram sorteados, de forma aleatória, 45 setores censitários na II PESN/PE (1997) e 39 setores censitários na III PESN/PE (2006). Finalmente no terceiro estágio foi adotado o critério do IBGE que considera, como ponto de referência em cada setor censitário selecionado, o ponto extremo 
da quadra voltada para o nascente, seguindo-se o sentido horário para a localização dos domicílios onde residiam crianças menores de cinco anos, até alcançar uma amostra em torno de quarenta crianças por cada setor censitário.

A amostra da II PESN foi recalculada para os fins específicos do presente estudo, tendo como referência a prevalência estimada de anemia de $36 \%$ para o Estado de Pernambuco, baseada no estudo de Monteiro et al. 3 , com um erro de 3,4\% e nível de confiança de $95 \%$ para a população total de crianças menores de cinco anos de idade no estado. Para o cálculo da amostra da III PESN, foi considerada uma prevalência de anemia de $40 \%$, erro de $3,1 \%$ e um nível de $95 \%$ de confiança 14 . No cálculo amostral das duas pesquisas foi adionada uma margem de $10 \%$ com o objetivo de compensar eventuais perdas.

Após a exclusão das crianças menores de seis meses para este estudo (não submetidos à coleta de sangue), as amostras totalizaram 777 e 993 crianças de 6-59 meses, nas II PESN e III PESN, respectivamente. Na redistribuição para compor os dois grupos etários, obtiveram-se amostras de 376 e 347 crianças de 6-23 meses e 401 e 646 crianças de 24-59 meses, respectivamente, para os inquéritos de 1997 e 2006.

As entrevistas foram realizadas com as mães ou outra pessoa responsável pela criança, utilizando formulários com dados referentes à identificação do domicílio, variáveis socioeconômicas, características da criança, antecedentes da gestação e morbidade, dados antropométricos e clínico-laboratoriais das mães e crianças.

No dia seguinte à entrevista foram coletadas amostras de sangue no laboratório ou no domicílio. A coleta de sangue foi realizada por punção venosa (por necessidade de outros exames para a pesquisa original) e utilizada uma gota de sangue para a análise de hemoglobina no equipamento Hemocue (Hemocue Limited, Sheffield, Reino Unido). O diagnóstico de anemia foi baseado nos padrões da OMS que considera anêmicas as crianças de 6-59 meses com concentração de hemoglobina abaixo de $11 \mathrm{~g} / \mathrm{dL}$, e as mulheres não gestantes em idade reprodutiva abaixo de $12 \mathrm{~g} / \mathrm{dL} 7$.

A avaliação antropométrica foi realizada segundo as recomendações da OMS 15. As crianças menores de dois anos foram pesadas descalças junto com as mães ou responsável, com vestimenta mínima, em balança digital (modelo MEA03200/Plenna. Plenner Equipamentos, Santo André, Brasil) com capacidade de $150 \mathrm{~kg}$ e escala de $100 \mathrm{~g}$, sendo o peso final obtido com a subtração dos pesos das respectivas mães.

A estatura em crianças de até dois anos foi medida utilizando-se um infantômetro (Altura- exata Ltda., Belo Horizonte, Brasil), com amplitude de $100 \mathrm{~cm}$ e precisão de $0,1 \mathrm{~cm}$, em decúbito dorsal. Nas maiores de dois anos foi usado um estadiômetro portátil confeccionado em coluna de madeira desmontável, com escala bilateral de 35 até $213 \mathrm{~cm}$ e precisão de $0,1 \mathrm{~cm}$. As crianças eram colocadas em posição ereta, descalças, com os membros superiores pendentes ao longo do corpo, os calcanhares, o dorso e a cabeça tocando a coluna de madeira.

Para avaliar o estado nutricional das crianças, foram utilizados três indicadores: peso para a idade, estatura para a idade e peso para a estatura, segundo a distribuição em escores Z. Para classificação das medidas antropométricas foi utilizado o padrão de referência para menores de cinco anos adotado pelo Ministério da Saúde 16. A avaliação antropométrica foi realizada usando-se o software Anthro, versão 3.01 (WHO Anthro 2009, Genebra, Suíça) adotando-se o seguinte critério: (a) indicador peso/idade: $<-2 \mathrm{SZ}=$ peso baixo ou peso muito baixo; $\geq-2 \mathrm{SZ}=$ peso adequado ou eutrófico; (b) indicador estatura/ idade: $<-2 \mathrm{SZ}=$ baixa estatura; $\geq-2 \mathrm{SZ}=$ estatura adequada; (c) indicador peso/estatura: $<-2 \mathrm{SZ}=$ peso baixo para a estatura; $\geq-2 S Z=$ peso adequado ou eutrófico.

Os fatores socioeconômicos analisados foram: área geográfica (urbana e rural), renda familiar em salários mínimos, escolaridade materna e trabalho remunerado materno. As variáveis referentes às condições do ambiente foram representadas por meio de um índice adaptado de Oliveira et al. 17. O índice ambiental foi composto pelas variáveis: destino dos dejetos; destino do lixo; origem da água; tratamento da água e condições de habitação, como tipo de piso, número de cômodos da casa e de pessoas que moravam no domicílio. Atribuiu-se o valor 0 para a situação desfavorável e 1 para a situação favorável. A soma desses valores foi categorizada em tercis, considerando-se o tercil 1 para a situação mais desfavorável, 2 para a intermediária e 3 para a situação mais favorável, caracterizando a condição ambiental dos domicílios.

O índice econômico foi composto pelas variáveis que indicam a disponibilidade de eletricidade na casa e a posse de bens de consumo (televisão, rádio, geladeira e fogão), atribuindose o valor 1 para cada um dos itens e 0 para a sua inexistência. A soma dos valores foi categorizada em tercis, caracterizando o índice econômico 17.

A utilização dos índices ambiental e econômico foi escolhida por estes reunirem as condições do ambiente sanitário do domicílio e da posse de bens de consumo. Os índices vêm sendo utilizados por estudos 6,18, uma vez que algumas variáveis testadas isoladamente não apresentam 
associação estatística com o problema, porém quando agrupadas em forma de índice observase uma significância estatística, aproximando-se da realidade do indivíduo e de suas condições ambientais e econômicas.

Em relação à assistência à saúde e nutrição, as variáveis estudadas foram a realização do pré-natal; número de consultas no pré-natal; e duração do aleitamento materno, categorizada em função do tempo legal de quatro meses da licença maternidade em vigor na época da coleta de dados.

As variáveis relacionadas à morbidade foram: a presença de diarreia no dia ou nos últimos 15 dias anteriores à entrevista e a presença de tosse no dia ou na semana anterior à entrevista. O fator biológico analisado foi o sexo da criança, figurando como fatores maternos a idade em anos e a anemia.

A evolução temporal da anemia nas faixas etárias foi analisada pela diferença das prevalências entre os inquéritos de 1997 e de 2006, e a sua significância estatística foi verificada pelo teste de qui-quadrado.

A análise bivariada foi realizada em cada pesquisa, segundo a faixa etária, utilizando-se a regressão de Poisson simples. As variáveis associadas com a anemia com valor de $\mathrm{p}<0,20$ foram selecionadas para compor o modelo de regressão múltipla.

As variáveis foram agrupadas em níveis hierárquicos de acordo com o modelo proposto por Osório et al. 14. Na concepção desse modelo, foi levada em consideração a ordenação de níveis, partindo dos determinantes distais para os determinantes proximais, assim distribuídos: no primeiro nível, as variáveis relacionadas aos fatores socioeconômicos; no segundo, aquelas referentes às condições de habitação, saneamento e posse de bens de consumo que foram agrupadas em índice econômico e índice ambiental; no terceiro, a condição materna e suas representações; no quarto, as variáveis ligadas à assistência à saúde e nutrição; e por fim, no quinto nível, as variáveis que expressam a morbidade e o estado nutricional.

O fator biológico avaliado foi o sexo da criança que seria utilizado para ajustar todo o modelo, porém, neste trabalho o sexo da criança não foi uma variável selecionada na análise bivariada, pois esta variável não apresentou um nível de significância de 0,20 , não compondo, portanto, os modelos explicativos em ambos os anos.

$\mathrm{Na}$ análise dos modelos de cada faixa etária foi utilizado o método de backward, considerando cada nível do modelo progressivamente, retirando-se a variável com valor de p superior às demais e com significância maior ou igual a $20 \%$, até que todas as variáveis apresentassem o valor de $\mathrm{p}<0,20$. Em seguida, adicionou-se a esse modelo as variáveis do 2 o nível hierárquico, procedendose, consecutivamente, da mesma maneira, com a exclusão progressiva das variáveis deste nível. Dessa forma, foram analisados todos os níveis hierárquicos. Após a finalização da análise de cada nível do modelo, as variáveis restantes permaneceram no processo de modelagem para ajuste do modelo final, no qual foram consideradas significantes as variáveis com $\mathrm{p}<0,05$.

Para o processamento dos dados foi utilizado o programa Epi Info versão 6,04 (Centers for Disease Control and Prevention, Atlanta, Estados Unidos), em dupla entrada dos dados. O programa R versão 2-15-1 (The R Foundation for Statistical Computing, Viena, Áustria) foi utilizado para as análises de Poisson simples e de regressão múltipla.

Os projetos da II PESN/PE (CEP/CCS/UFPE, 27/02/1997) e da III PESN/PE (processo no 1321, 2004) foram aprovados pelo Comitê de Ética em Pesquisa em Seres Humanos do IMIP, configurando-se, na prática, com a assinatura do termo de consentimento livre e esclarecido do responsável pela criança. Os casos de anemia receberam tratamento medicamentoso com sulfato ferroso oral e foram encaminhados para acompanhamento adequado no serviço de saúde mais acessível a cada caso pessoal.

\section{Resultados}

A média de idade das crianças examinadas em 1997 foi 28,1 meses, com desvio-padrão (DP) de 15,4 meses. Já em 2006, a média de idade foi de $31,6$ meses ( $\mathrm{DP}=15,7)$.

Entre as crianças de 6-23 meses ocorreu uma redução da prevalência de anemia de $63 \%$ para $55,6 \%\left(\chi^{2}=4,11, p=0,04\right)$, ou seja, uma queda de $11,7 \%$. Para as crianças de $24-59$ meses as prevalências observadas nos anos de 1997 e 2006 foram, respectivamente, de $31,4 \%$ e $20,9 \%$, representando, portanto, uma diminuição de $33,4 \%$ $\left(\chi^{2}=14,64, \mathrm{p}<0,001\right)$.

As variáveis incluídas na primeira etapa (screening) da análise de regressão para as crianças de 6-23 meses nos anos de 1997 e 2006 foram semelhantes (Tabela 1), com exceção das variáveis peso ao nascer e idade da mãe, selecionadas apenas para compor o modelo em 1997. Por não ter sido contemplada na pesquisa de 1997 , a prevalência de anemia materna não entrou no modelo, apesar de ter apresentado o nível de significância de $<$ 0,05 em 2006 tanto para as crianças de 6-23 como para as de 24-59 meses. 
Prevalência de anemia em crianças de 6-23 meses, segundo estado nutricional, fatores socioeconômicos, índice econômico, índice ambiental, fatores maternos e assistência à saúde. Pernambuco, Brasil, 1997, 2006.

\begin{tabular}{|c|c|c|c|c|c|c|c|c|}
\hline \multirow[t]{2}{*}{ Variáveis } & \multicolumn{4}{|c|}{ II PESN/PE $1997(n=376)$} & \multicolumn{4}{|c|}{ III PESN/PE $2006(n=347)$} \\
\hline & $\mathbf{n}$ & $\%$ & RP (IC95\%) & Valor de $p$ & $\mathbf{n}$ & $\%$ & RP (IC95\%) & Valor de $p$ \\
\hline \multicolumn{9}{|c|}{ Área geográfica } \\
\hline Rural & 127 & 70,9 & $0,84(0,72 ; 0,97)$ & 0,02 & 96 & 68,8 & $1,36(1,13 ; 1,63)$ & $<0,001$ \\
\hline Urbana & 249 & 59,0 & 1,00 & & 251 & 50,6 & 1,00 & \\
\hline \multicolumn{9}{|c|}{ Renda familiar (salários mínimos) } \\
\hline$<2$ & 170 & 71,2 & $1,27(1,09 ; 1,49)$ & $<0,001$ & 244 & 60,2 & $1,3(1,03 ; 1,68)$ & 0,03 \\
\hline$\geq 2$ & 202 & 55,9 & 1,00 & & 94 & 45,7 & 1 & \\
\hline \multicolumn{9}{|c|}{ Escolaridade materna (anos) } \\
\hline $0-8$ & 309 & 66,3 & $1,40(1,07 ; 1,80)$ & 0,01 & 251 & 61,4 & $1,50(1,15 ; 1,93)$ & $<0,001$ \\
\hline$\geq 9$ & 67 & 47,8 & 1,00 & & 95 & 41,1 & 1,00 & \\
\hline \multicolumn{9}{|c|}{ Índice econômico (tercil) } \\
\hline 1 & 114 & 68,4 & $1,23(1,03 ; 1,48)$ & & 69 & 71,0 & $1,52(1,23 ; 1,90)$ & \\
\hline 2 & 87 & 71,3 & $1,28(1,06 ; 1,55)$ & & 93 & 62,4 & $1,34(1,07 ; 1,67)$ & \\
\hline 3 & 175 & 55,4 & 1,00 & 0,02 & 185 & 46,5 & 1,00 & $<0,001$ \\
\hline \multicolumn{9}{|c|}{ Índice ambiental (tercil) } \\
\hline 1 & 114 & 78,1 & $1,45(1,20 ; 1,75)$ & & 134 & 69,4 & $2,51(1,70 ; 3,74)$ & \\
\hline 2 & 136 & 58,8 & $1,09(0,88 ; 1,35)$ & & 134 & 56,7 & $2,05(1,36 ; 3,10)$ & \\
\hline 3 & 126 & 54,0 & 1,00 & $<0,001$ & 69 & 27,5 & 1 & $<0,001$ \\
\hline \multicolumn{9}{|c|}{ Idade da mãe (anos) } \\
\hline$<20$ & 69 & 55,1 & $0,90(0,68 ; 1,07)$ & 0,17 & 61 & 60,7 & $1,12(0,89 ; 1,39)$ & 0,36 \\
\hline$\geq 20$ & 306 & 64,7 & 1,00 & & 286 & 54,5 & 1,00 & \\
\hline \multicolumn{9}{|c|}{ Anemia materna } \\
\hline Presente & & & & & 71 & 69,0 & $1,31(1,07 ; 1,58)$ & 0,01 \\
\hline Ausente & & & & & 254 & 52,8 & 1,00 & \\
\hline \multicolumn{9}{|c|}{ Número de consultas pré-natal } \\
\hline$\leq 5$ & 210 & 66,7 & $1,20(0,99 ; 1,38)$ & 0,06 & 88 & 67,0 & $1,30(1,06 ; 1,55)$ & 0,01 \\
\hline$\geq 6$ & 158 & 57,0 & 1,00 & & 243 & 52,3 & 1,00 & \\
\hline \multicolumn{9}{|c|}{ Peso ao nascer (g) } \\
\hline$<2.500$ & 27 & 74,1 & $1,20(0,95 ; 1,54)$ & 0,12 & 26 & 65,4 & $1,20(0,88 ; 1,60)$ & 0,27 \\
\hline$\geq 2.500$ & 327 & 61,2 & 1,00 & & 315 & 55,2 & 1,00 & \\
\hline \multicolumn{9}{|c|}{ Índice estatura/idade } \\
\hline$<-2 S Z$ & 66 & 81,8 & $1,39(1,21 ; 1,62)$ & $<0,001$ & 23 & 54,6 & $1,27(0,95 ; 1,70)$ & 0,10 \\
\hline$\geq-2 S Z$ & 307 & 58,6 & 1,00 & & 324 & 69,6 & 1,00 & \\
\hline
\end{tabular}

IC95\%: intervalo de 95\% de confiança; II PESN/PE: II Pesquisa Etadual de Saúde e Nutrição de Pernambuco; III PESN/PE: III Pesquisa Etadual de Saúde e Nutrição de Pernambuco; RP: razão de prevalência.

Para as crianças com idades entre 24-59 meses, os resultados das variáveis selecionadas para a análise múltipla se modificaram, não se repetindo nos modelos de 1997 e 2006 (Tabela 2), sendo o índice econômico a única variável que se manteve nos dois modelos.

No inquérito de 1997 em crianças de 6-23 meses, as variáveis que permaneceram significativas na análise de regressão múltipla foram a renda familiar (nível 1) e o índice ambiental (nível 2), após os ajustes pela escolaridade materna, idade da mãe e peso ao nascer. Nessa faixa etária, no estudo em 2006, foram mantidos no modelo final a área geográfica e a escolaridade materna no nível 1 e o índice ambiental no nível 2, depois de ajuste pelo índice econômico e número de consultas do pré-natal (Tabela 3 ).

Entre as crianças de 24-59 meses, as variáveis significativas no modelo final de regressão múltipla em 1997 foram a área geográfica no nível 1, e no nível 2 permaneceram o índice econômico e o índice ambiental e, finalmente no nível 3, a 
Tabela 2

Prevalência de anemia em crianças de 24-59 meses, segundo morbidade, estado nutricional, fatores socioeconômicos, índice econômico, índice ambiental, fatores maternos e assistência à saúde. Pernambuco, Brasil, 1997, 2006.

\begin{tabular}{|c|c|c|c|c|c|c|c|c|}
\hline \multirow[t]{2}{*}{ Variáveis } & \multicolumn{4}{|c|}{ II PESN/PE $1997(n=401)$} & \multicolumn{4}{|c|}{ III PESN/PE 2006 ( $n=646)$} \\
\hline & $\mathrm{n}$ & $\%$ & RP (IC95\%) & Valor de $\mathrm{p}$ & $\mathrm{n}$ & $\%$ & RP (IC95\%) & Valor de $p$ \\
\hline \multicolumn{9}{|c|}{ Área geográfica } \\
\hline Rural & 126 & 41,3 & $1,54(1,15 ; 2,03)$ & $<0,001$ & 180 & 20,0 & $0,94(0,67 ; 1,32)$ & 0,73 \\
\hline Urbana & 275 & 26,9 & 1,00 & & 466 & 21,2 & 1,00 & \\
\hline \multicolumn{9}{|c|}{ Renda familiar (salários mínimos) } \\
\hline$<2$ & 172 & 37,2 & $1,38(1,03 ; 1,84)$ & 0,03 & 436 & 21,8 & $1,20(0,84 ; 1,65)$ & 0,35 \\
\hline$\geq 2$ & 225 & 27,1 & 1,00 & & 200 & 18,5 & 1,00 & \\
\hline \multicolumn{9}{|c|}{ Escolaridade materna (anos) } \\
\hline $0-8$ & 324 & 34,3 & $1,73(1,07 ; 2,80)$ & 0,02 & 468 & 21,6 & $1,10(0,79 ; 1,62)$ & 0,49 \\
\hline$\geq 9$ & 76 & 19,7 & 1,00 & & 173 & 19,1 & 1,00 & \\
\hline \multicolumn{9}{|c|}{ Trabalho materno remunerado } \\
\hline Não & 274 & 32,8 & $1,20(0,86 ; 1,67)$ & 0,29 & 465 & 22,8 & $1,40(0,97 ; 2,03)$ & 0,07 \\
\hline $\operatorname{Sim}$ & 124 & 27,4 & 1,00 & & 179 & 16,2 & 1,00 & \\
\hline \multicolumn{9}{|c|}{ Índice econômico (tercil) } \\
\hline 1 & 107 & 48,6 & $2,25(1,63 ; 3,13)$ & & 102 & 32,4 & $1,70(1,20 ; 2,41)$ & \\
\hline 2 & 90 & 33,3 & $1,55(1,04 ; 2,29)$ & & 156 & 17,9 & $0,94(0,64 ; 1,39)$ & \\
\hline 3 & 204 & 21,6 & 1,00 & $<0,001$ & 388 & 19,1 & 1,00 & 0,02 \\
\hline \multicolumn{9}{|c|}{ Índice ambiental (tercil) } \\
\hline 1 & 120 & 50,0 & $2,34(1,65 ; 3,35)$ & & 252 & 22,6 & $1,25(0,81 ; 1,93)$ & \\
\hline 2 & 131 & 26,0 & $1,22(0,79 ; 1,86)$ & & 256 & 21,1 & $1,16(0,75 ; 1,80)$ & \\
\hline 3 & 150 & 21,3 & 1,00 & $<0,001$ & 127 & 18,1 & 1,00 & 0,32 \\
\hline \multicolumn{9}{|c|}{ Idade da mãe (anos) } \\
\hline$<20$ & 30 & 33,3 & $1,10(0,63 ; 1,82)$ & 0,80 & 42 & 33,3 & $1,67(1,05 ; 2,61)$ & 0,03 \\
\hline$\geq 20$ & 369 & 31,2 & 1,00 & & 603 & 20,1 & 1,00 & \\
\hline \multicolumn{9}{|c|}{ Anemia materna } \\
\hline Presente & & & & & 114 & 34,2 & $1,86(1,36 ; 2,56)$ & $<0,001$ \\
\hline Ausente & & & & & 475 & 18,3 & 1,00 & \\
\hline \multicolumn{9}{|l|}{ Diarreia } \\
\hline Sim & 60 & 43,3 & $1,46(1,05 ; 2,05)$ & 0,02 & 97 & 20,9 & $1,00(0,64 ; 1,51)$ & 0,94 \\
\hline Não & 339 & 29,5 & 1,00 & & 549 & 20,6 & 1,00 & \\
\hline \multicolumn{9}{|c|}{ Índice estatura/idade } \\
\hline$<-2 S Z$ & 59 & 37,3 & $1,23(0,85 ; 1,79)$ & 0,27 & 46 & 19,8 & $1,42(0,88 ; 2,32)$ & 0,15 \\
\hline$\geq-2 S Z$ & 337 & 30,3 & 1,00 & & 590 & 28,3 & 1,00 & \\
\hline
\end{tabular}

IC95\%: intervalo de 95\% de confiança; II PESN/PE: II Pesquisa Etadual de Saúde e Nutrição de Pernambuco; III PESN/PE: III Pesquisa Etadual de Saúde e Nutrição de Pernambuco; RP: razão de prevalência.

ocorrência de diarreia, após ajustamento pela escolaridade materna. Em 2006, as variáveis significativas foram o índice econômico no nível 2 e a idade da mãe no nível 3, ajustadas no modelo pela variável trabalho materno remunerado (Tabela 4).

As variáveis explicativas da anemia diferiram nas pesquisas de 1997 e 2006 nos modelos de análise multivariada. Para as crianças com idades entre 6-23 meses, apenas o índice ambiental permaneceu como fator determinante da ane- mia nos anos de 1997 e 2006. Nas crianças de 24-59 meses, na comparação da tendência temporal, somente o índice econômico permaneceu associado à anemia.

\section{Discussão}

Torna-se relevante observar que, de fato, ocorreu uma evolução favorável no quadro epidemiológico, evidenciada na redução de $11,7 \%$ das ane- 
Razões de prevalência (RP) ajustadas da anemia em crianças de 6-23 meses, dados das II e III Pesquisa Estadual de Saúde e Nutrição de Pernambuco (PESN/PE) em 1997 e 2006, respectivamente.

\begin{tabular}{|c|c|c|c|c|}
\hline \multirow[t]{2}{*}{ Nível/Variáveis } & \multicolumn{2}{|c|}{ II PESN/PE $1997(n=376)$ * } & \multicolumn{2}{|c|}{ III PESN/PE $2006(n=347)$ ** } \\
\hline & RP (IC95\%) & Valor de $p$ & RP (IC95\%) & Valor de $p$ \\
\hline \multicolumn{5}{|l|}{ Nível 1} \\
\hline \multicolumn{5}{|c|}{ Área geográfica } \\
\hline Rural & & & $1,03(1,05 ; 1,52)$ & \\
\hline Urbana & & & 1,00 (Referência) & 0,014 \\
\hline \multicolumn{5}{|c|}{ Renda familiar (salários mínimos) } \\
\hline$<2$ & $1,20(1,03 ; 1,42)$ & & & \\
\hline$\geq 2$ & 1,00 (Referência) & 0,021 & & \\
\hline \multicolumn{5}{|c|}{ Escolaridade materna (anos) } \\
\hline $0-8$ & & & $1,40(1,09 ; 1,84)$ & \\
\hline$\geq 9$ & & & 1,00 (Referência) & 0,010 \\
\hline \multicolumn{5}{|l|}{ Nível 2} \\
\hline \multicolumn{5}{|c|}{ Índice ambiental (tercil) } \\
\hline 1 & $1,03(1,02 ; 1,57)$ & & $2,10(1,39 ; 3,33)$ & \\
\hline 2 & $1,00(0,79 ; 1,25)$ & & $2,00(1,30 ; 2,97)$ & \\
\hline 3 & 1,00 (Referência) & 0,015 & 1,00 (Referência) & 0,000 \\
\hline
\end{tabular}

IC95\%: intervalo de 95\% de confiança.

* Ajustadas pelas variáveis escolaridade materna, idade da mãe e peso ao nascer;

** Ajustada pelas variáveis índice econômico e número de consultas pré-natal.

mias em crianças de 6-23 meses entre 1997 e 2006 e, principalmente, na redução de $33,4 \%$ na faixa de 24-59 meses. Em outros termos, a redução de $19,3 \%$ para o conjunto de menores de cinco anos deve-se, em sua maior parte, ao impacto da mudança ocorrida nas crianças de 24-59 meses 6 .

Em nível nacional, embora não seja um consenso, existem seguras evidências de que o panorama epidemiológico referente à anemia, sobretudo em crianças, passou a melhorar após 2004, quando o Ministério da Saúde tornou obrigatório o enriquecimento de massas alimentares de elevado consumo (trigo e milho) com a adição de ferro e folato ${ }^{19}$. Sugere-se que o impacto desse programa tenha sido mais favorável às crianças maiores em virtude de uma quantidade mais elevada do consumo desses alimentos enriquecidos 8,9. Contrapondo-se a essa hipótese, os estudos de Pelotas, Rio Grande do Sul 20,21, não observaram diferença significativa entre os momentos antes e após a fortificação obrigatória das farinhas em crianças de 12 a 24 meses. No entanto, como o Brasil é um país de vasta extensão geográfica e diversidades culturais, econômicas e sociais, a necessidade de outros estudos é inegável para avaliar de fato o efeito da suplementação das farinhas em nível nacional.
Outro fator que poderia explicar a maior redução na faixa etária de 24-59 meses é a probabilidade do grupo atingir mais facilmente o ponto de corte de $11 \mathrm{~g} / \mathrm{dL}$ estabelecido pela OMS 7 como descriminante da anemia, visto que os níveis de hemoglobina nas crianças descrevem, fisiologicamente, uma curva em "U", ou seja, valores altos no nascimento rebaixados em torno de 6-11 meses, elevando-se progressivamente até a puberdade 22 .

Em contraposição aos dados do presente estudo, no México houve uma tendência diferente, observando-se uma maior diminuição da prevalência entre os menores de dois anos, o que se deve, possivelmente, ao fato de que este era o grupo alvo do programa de intervenção Oportunidades. Entre outras ações, esse programa realizava a distribuição de alimento fortificado com ferro, zinco e outros micronutrientes para crianças de 6-24 meses a partir de 199923 .

Ordinariamente no presente estudo, as crianças menores de 24 meses apresentam maiores prevalências de anemia em relação às maiores de 24 meses. Essa característica pode ser devida às necessidades de ferro particularmente elevadas, em função do crescimento corporal acelerado naquela faixa etária, além do desmame precoce e da oferta de uma alimentação monótona e pobre 
Razões de prevalência (RP) ajustadas da anemia em crianças de 24-59 meses, dados das II e III Pesquisa Estadual de Saúde e Nutrição de Pernambuco (PESN/PE) em 1997 e 2006, respectivamente.

\begin{tabular}{|c|c|c|c|c|}
\hline \multirow[t]{2}{*}{ Nível/Variáveis } & $\begin{array}{l}\text { II PESN/PE } 1997 \\
(n=401) \text { * }\end{array}$ & Valor de $p$ & $\begin{array}{l}\text { III PESN/PE } 2006 \\
\quad(n=646) * \star\end{array}$ & Valor de $p$ \\
\hline & RP (IC95\%) & & RP (IC95\%) & \\
\hline \multicolumn{5}{|l|}{ Nível 1} \\
\hline \multicolumn{5}{|c|}{ Área geográfica } \\
\hline Rural & $1,40(1,05 ; 1,88)$ & & & \\
\hline Urbana & 1,00 (Referência) & 0,022 & & \\
\hline \multicolumn{5}{|l|}{ Nível 2} \\
\hline \multicolumn{5}{|c|}{ Índice econômico (tercil) } \\
\hline 1 & $1,70(1,14 ; 2,65)$ & & $1,60(1,14 ; 2,31)$ & \\
\hline 2 & $1,30(0,84 ; 1,94)$ & 0,010 & $0,90(0,61 ; 1,34)$ & 0,03 \\
\hline 3 & 1,00 (Referência) & & 1,00 (Referência) & \\
\hline \multicolumn{5}{|c|}{ Índice ambiental (tercil) } \\
\hline 1 & $1,80(1,10 ; 2,83)$ & & & \\
\hline 2 & $1,00(0,64 ; 1,59)$ & & & \\
\hline 3 & 1,00 (Referência) & 0,009 & & \\
\hline \multicolumn{5}{|l|}{ Nível 3} \\
\hline \multicolumn{5}{|c|}{ Idade da mãe (anos) } \\
\hline$<20$ & & & $1,60(1,00 ; 2,52)$ & \\
\hline$\geq 20$ & & & 1,00 (Referência) & 0,04 \\
\hline \multicolumn{5}{|l|}{ Diarreia } \\
\hline Sim & $1,40(1,03 ; 1,95)$ & & & \\
\hline Não & 1,00 (Referência) & 0,033 & & \\
\hline
\end{tabular}

IC95\%: intervalo de 95\% de confiança.

* Ajustada pela variável escolaridade materna;

** Ajustada pela variável trabalho materno remunerado.

em alimentos ricos em ferro que se segue à transição de uma dieta predominantemente láctea para uma alimentação mais diversificada 24,25.

A mesma explicação poderia ser atribuída ao maior risco de anemia nas crianças de 6-23 meses que residiam na área rural no ano de 2006, em contraste com crianças de 24-59 meses, grupo que apresentou maior diminuição da prevalência de anemia. $\mathrm{O}$ estudo realizado no Município de Jordão, no Acre, é particularmente elucidativo, observando-se $37,5 \%$ de anemia nas crianças com ascendência indígena da área urbana $\mathrm{e}$ uma prevalência bastante superior $(64,3 \%)$ nas crianças da área rural 26. Já a Pesquisa Nacional de Demografia e Saúde de 2006 (PNDS/2006) constatou uma relação estatística bem diferente, observando-se que as crianças de áreas rurais apresentaram uma menor prevalência de anemia quando comparadas às crianças de áreas urbanas 27 .

Na caracterização das habitações, alguns estudos verificaram maior prevalência de ane- mia em crianças que viviam em casas com acabamento irregular, alta aglomeração de pessoas por cômodo e em domicílios que possuíam sanitário sem descarga 28,29. Na análise bivariada aqui relatada, os índices ambiental e econômico foram associados à anemia nas crianças de 6-23 meses em 1997 e 2006, embora apenas o índice ambiental tenha se mantido significativo no modelo multivariado nos anos estudados.

O aumento da renda, atribuído cumulativamente aos programas de transferência de renda, ao aumento do salário mínimo, ao acréscimo dos vales-refeições, aos custos implícitos das ações de saúde disponibilizadas pelo processo de desenvolvimento econômico que o país tem vivenciado levaram a alterações dos fatores determinantes da anemia 3 . No caso de Pernambuco observa-se que a renda familiar perdeu o seu valor explicativo no modelo multivariado aplicado às crianças de 6-23 meses em 2006, enquanto outro fator, a escolaridade materna, foi relativamente mais importante. 
As estratégias de transferência de renda reunidas no Programa Bolsa Família em 2003 têm impactado na elevação da renda per capita, de modo que a situação das famílias beneficiadas e a das famílias não beneficiadas pelo programa tende a se aproximar estatisticamente 29 .

As famílias beneficiadas pelo Programa Bolsa Família utilizam grande parte do recurso para a compra de alimentos, o que tem um impacto positivo na quantidade e qualidade da alimentação das famílias ${ }^{30}$. No entanto, as condições sanitárias e de moradia permanecem precárias, e estas são amplamente descritas como variáveis preditivas da anemia. É inegável o impacto positivo do Programa Bolsa Família na qualidade de vida dos indivíduos. Mas, ressalta-se a importância de ações estruturantes que melhorem as condições de moradia e viabilizem o saneamento básico nas populações carentes 29,30,31.

Nas crianças de 24-59 meses, apenas o índice econômico, e não a renda, se confirmou na regressão logística, representando uma variável que permaneceu associada à anemia nos anos de 1997 e 2006. Assim, esses resultados indicam que as crianças maiores parecem ser mais sensíveis às características econômicas refletidas na posse de bens de consumo do que à variação da renda familiar.

A ocorrência de diarreia nos 15 dias anteriores à pesquisa foi associada à anemia quando avaliada isoladamente na análise bivariada e em conjunto com os outros fatores determinantes no modelo multivariado nas crianças de 24-59 meses em 1997, mas esta associação não se manteve em 2006 quando, inclusive, a ocorrência de diarreia não foi selecionada para participar do modelo desse ano. Sabe-se que existe uma marcante predisposição ao surgimento da anemia após um episódio de infecção aguda, como as diarreias, mas seus efeitos passam a depender da duração e severidade do processo para resultar um efeito detectável sobre o risco de anemia 31. É pertinente considerar que a implantação da Estratégia Saúde da Família (ESF) representa uma melhora sensível na situação de saúde dos indivíduos, possibilitando evitar e/ou minimizar episódios de infecção aguda 31,32.

Outra condição que pode ter contribuído para reduzir o problema dos agravos infecciosos, incluindo as diarreias, foi o acompanhamento das crianças pelo cumprimento das ações básicas de saúde, como condicionalidade para a manutenção do benefício do Programa Bolsa Família 29.

Como observação genérica, é oportuno assinalar no presente estudo a falta de informações sobre a situação alimentar de crianças menores de cinco anos. Como o valor nutricional da dieta é uma chave explicativa de marcante importância para a análise do problema de anemia, a inexistência de informações sobre a quantidade, qualidade (biodisponibilidade) e fatores facilitadores ou inibidores do aproveitamento de ferro, como a vitamina $\mathrm{C}$ e as proteínas de origem animal, constitui limitações para se entender a razão das diferenças nos fatores explicativos do problema nos dois grupos etários.

Uma evidência indireta nesse sentido seria representada pela avaliação de Silva et al. 33, que estudaram a prevalência e fatores de risco de anemia em mães e filhos menores de cinco anos em Pernambuco, demonstrando que, a partir dos três anos de idade, o problema em mães e filhos passa a se equivaler. Seria o caso de se admitir que a alimentação das crianças passaria a corresponder a alimentação das mães ou seus familiares, diferindo assim do que acontece com crianças de mais baixa idade, quando os leites e as massas alimentares são mais utilizados. É uma hipótese que pode e deve ser aprofundada.

A idade da mãe tem se mostrado estatisticamente associada à prevalência de anemia em outros estudos populacionais 5,33. A associação da anemia dos filhos de mães mais jovens provavelmente se deve à maior probabilidade de conceberem filhos com baixo peso, bem como da inexperiência materna nos cuidados com as crianças, que tendem a apresentar maiores dificuldades de alimentar adequadamente seus filhos.

Em síntese, sob o aspecto conceitual e de ações estratégicas, é importante notificar que, entre 1997 a 2006, no Estado de Pernambuco, duas constatações ficam bem evidenciadas: a redução da prevalência das anemias em crianças como uma nova tendência epidemiológica demarcando diferença entre as faixas etárias de 6-23 meses e de 24-59 meses e mais, bem como os fatores de sua determinação nos dois grupos comparados. Dessa maneira, é importante configurar que as ações de intervenção para a redução do problema devem considerar os efeitos de diferentes interações enfocadas no presente estudo. 


\section{Resumen}

Para analizar la tendencia temporal de la anemia y sus factores asociados en niños de 6-23 y 24-59 meses en el estado de Pernambuco, Brasil, se utilizaron datos de dos encuestas de salud y nutrición. Las muestras sumaron 777 y 993 niños de 6 y 59 meses, en II PESN/1997 y III PESN/2006, respectivamente. Las variables explicativas se agruparon en cinco niveles jerárquicos: factores socioeconómicos; índice ambiental y económico; factores maternos; salud y nutrición; morbilidad y estado nutricional; analizado mediante regresión múltiple de Poisson. Entre las dos encuestas, la prevalencia de anemia es una disminución de 11,7\% y 33,4\%, respectivamente, para niños de 6-23 y 24-59 meses. En el análisis de tendencias en el tiempo, sólo el tercio inferior del indice ambiental permaneció como determinante de anemia para los niños 6-23 meses de edad, mientras que el tercio inferior del índice económico se mantuvo estadísticamente significativo en niños de 24-59 meses. Se concluyó que había más disminución significativa de anemia en el grupo de 24-59 meses.

Estudios de Series Temporales; Anemia; Niño

\section{Colaboradores}

P. N. Vasconcelos, L. P. Leal, M. M. Osório e M. Batista Filho participaram na concepção do projeto, análise e interpretação dos dados, redação e revisão final do manuscrito. D. S. Cavalcanti colaborou na análise e interpretação dos dados, redação e revisão final do manuscrito.

\section{Agradecimentos}

Ao CNPq pelo apoio financeiro.

\section{Referências}

1. Batista Filho M, Souza AI, Miglioli TC, Santos MC. Anemia e obesidade: um paradoxo da transição nutricional brasileira. Cad Saúde Pública 2008 24:247-57.

2. Oliveira RS, Diniz AS, Benigna MJC, Miranda-Silva SM, Lola MM, Gonçalves MC, et al. Magnitude, distribuição espacial e tendência da anemia em pré-escolares da Paraíba. Rev Saúde Pública 2002; 36:26-32.

3. Monteiro CA, Szarfarc SC, Mondini L. Tendência secular da anemia na infância na Cidade de São Paulo (1984-1996). Rev Saúde Pública 2000; 34(6 Suppl):62-72.

4. Vieira RCS, Ferreira HS. Prevalência de anemia em crianças brasileiras, segundo diferentes cenários epidemiológicos Rev Nutr 2010; 23:433-44.

5. Leal LP, Batista Filho M, Lira PIC, Figueiroa JN, Osório MM. Prevalência da anemia e fatores associados em crianças de seis a 59 meses de Pernambuco. Rev Saúde Pública 2011; 45:457-66.

6. LealLP,BatistaFilho M, LiraPIC, FigueiroaJN, Osório MO. Temporal trends and anaemia-associated factors in 6- to 59-month-old children in Northeast Brazil. Public Health Nutr 2012; 15:1645-52.
7. World Health Organization. Worldwide prevalence of anaemia 1993-2005: WHO Global Database on Anaemia. Geneva: World Health Organization; 2008.

8. Fujimori E, Sato APS, Szarfarc SC, Veiga GV, Oliveira VA, Colli C, et al. Anemia em gestantes brasileiras antes e após a fortificação de farinhas com ferro. Rev Saúde Pública 2011; 45:1027-35.

9. Costa CA, Machado EH, Colli C, Latorre WC, Szarfarc SC. Anemia em pré-escolares atendidos em creches de São Paulo (SP): perspectivas decorrentes das fortificações de farinhas de trigo e de milho. Nutrire Rev Soc Bras Aliment Nutr 2009; 34:59-74.

10. Oliveira MAA, Osório MM, Raposo MCF. Fatores socioeconômicos e dietéticos de risco para a anemia em crianças de 6 a 59 meses de idade. J Pediatr (Rio J.) 2007; 83:39-46.

11. Ministério da Saúde. II Pesquisa Estadual de Saúde e Nutrição: saúde, nutrição, alimentação e condições socioeconômicas no Estado de Pernambuco. Recife: Instituto Nacional de Alimentação e Nutrição; 1998 . 
12. Departamento de Nutrição, Centro de Ciências da Saúde, Universidade Federal de Pernambuco. III Pesquisa Estadual de Saúde e Nutrição: saúde, nutrição, alimentação e condições socioeconômicas e atenção à saúde no Estado de Pernambuco. I Inquérito estadual sobre doenças crônicas e agravos não transmissíveis: prevalência e fatores de risco. Recife: Universidade Federal de Pernambuco; 2012

13. Governo de Pernambuco; Fundo das Nações Unidas para a Infância. Crianças e adolescentes em Pernambuco; saúde, educação e trabalho. Recife: Governo de Pernambuco/Brasília: Fundo das Nações Unidas para a Infância; 1992.

14. Osório MM, Lira PIC, Ashworth A. Factors associated with $\mathrm{Hb}$ concentration in children aged 6-59 months in the State of Pernambuco, Brazil. Br J Nutr 2004; 91:307-14.

15. WHO Working Group on Infant Growth. An evaluation of infant growth: the use and interpretation of anthropometry in infants. Bull World Health Organ 1995; 73:165-74.

16. Multicentre Growth Reference Study Group, World Health Organization. WHO Child Growth Standards: length/height-for-age, weight-forage weight-for-length, weight-for-height and body mass index-for-age. Methods and development. Geneva: World Health Organization; 2006.

17. Oliveira LPM, Barreto ML, Assis AMO, Braga-Junior ACR, Nunes MFFP, Oliveira NF, et al. Preditores do retardo de crescimento linear em préescolares: uma abordagem multinível. Cad Saúde Pública 2007; 23:601-13.

18. Issler RM, Giugliani ERJ. Identificação de grupos mais vulneráveis à desnutrição infantil pela medição do nível de pobreza. J Pediatr (Rio J.) 1997; 73:101-5.

19. Agência Nacional de Vigilância Sanitária. Resolução RDC no 344, de 13 de dezembro de 2002. Regulamento técnico para fortificação das farinhas de trigo e das farinhas de milho com ferro e ácido fólico. Diário Oficial da União 2002; $18 \mathrm{dez}$.

20. Assunção MCF, Santos IS, Barros AJD, Gigante DP, Victora CG. Flour fortification with iron has no impact on anaemia in urban Brazilian children. Public Health Nutr 2012; 15:1796-801.

21. Assunção MCF, Santos IS, Barros AJD, Gigante DP, Victora CG. Efeito da fortificação de farinha com ferro sobre anemia em pré-escolares, Pelotas, RS. Rev Saúde Pública 2007; 41:539-48.

22. Failace R. Hemograma: manual de interpretação. 5a Ed. Porto Alegre: Editora Artmed; 2009.
23. Villalpando S, Shamah-Levy T, García-Guerra A, Mundo-Rosas V, Domínguez C, Mejía-Rodríguez F. The prevalence of anemia decreased in Mexican preschool and school-age children from 1999 to 2006. Salud Pública Méx 2009; 51 Suppl 4:S507-14.

24. Lozoff B, Kaciroti N, Walter T. Iron deficiency in infancy: applying a physiologic framework for prediction. Am J Clin Nutr 2006; 84:1412-21.

25. Gondim SSR, Diniz AS, Souto RA, Bezerra RGS, Albuquerque EC, Paiva AA. Magnitude, tendência temporal e fatores associados à anemia em crianças do Estado da Paraíba. Rev Saúde Pública 2012; 46:649-56

26. Oliveira CS, Cardoso MA, Araújo TS, Muniz PT. Anemia em crianças de 6 a 59 meses e fatores associados no Município de Jordão, Estado do Acre, Brasil. Cad Saúde Pública 2011; 27:1008-20.

27. Ministério da Saúde. Pesquisa Nacional de Demografia e Saúde da Criança e da Mulher - PNDS 2006: dimensões do processo reprodutivo e da saúde da criança. Brasília: Ministério da Saúde; 2009.

28. Cotta RMM, Oliveira FCC, Magalhães KA, Ribeiro AQ, Sant'Ana LFR, Priore SE, et al. Social and biological determinants of iron deficiency anemia. Cad Saúde Pública 2011; 27 Suppl 2:S309-20.

29. Oliveira FCC, Cotta RMM, Ribeiro AQ, Sant'Ana LFR, Priore SE, Franceschini SCC. Estado nutricional e fatores determinantes do déficit estatural em crianças cadastradas no Programa Bolsa Família. Epidemiol Serv Saúde 2011; 20:7-18.

30. Duarte GB, Sampaio B, Sampaio Y. Programa Bolsa Família: impacto das transferências sobre os gastos com alimentos em famílias rurais. Revista de Economia e Sociologia Rural 2009; 47:903-18.

31. Roncalli AG, Lima KC. Impacto do Programa Saúde da Família sobre indicadores de saúde da criança em municípios de grande porte da região Nordeste do Brasil. Ciênc Saúde Coletiva 2006; 11:713-24.

32. Giovanella L, Mendonça MHM, Almeida PF, Escorel S, Senna MCM, Fausto MCR, et al. Saúde da família: limites e possibilidades para uma abordagem integral de atenção primária à saúde no Brasil. Ciênc Saúde Coletiva 2009; 14:783-94.

33. Silva SCL, Batista Filho M, Miglioli TC. Prevalência e fatores de risco de anemia em mães e filhos no Estado de Pernambuco. Rev Bras Epidemiol 2008; 11:266-77.

Recebido em 18/Jun/2013

Versão final reapresentada em 14/Dez/2013

Aprovado em 10/Fev/2014 